\title{
The metallic sphere in a uniform ac magnetic field: A simple and precise experiment for exploring eddy currents and non-destructive testing
}

\author{
Michael L. Honke ${ }^{\text {a) }}$ and Christopher P. Bidinosti ${ }^{\mathrm{b})}$ \\ Department of Physics, University of Winnipeg, 515 Portage Avenue, Winnipeg, Manitoba, Canada R3B 2E9
}

(Received 1 September 2017; accepted 8 April 2018)

\begin{abstract}
We describe a very simple experiment that utilizes standard laboratory equipment to measure the electromagnetic response of a metallic sphere exposed to a uniform ac magnetic field. Measurements were made for a variety of non-magnetic and magnetic metals, and in all cases the results fit very well with theory over the four orders of frequency $(25 \mathrm{~Hz}$ to $102 \mathrm{kHz})$ explored here. Precise values of magnetic permeability and electrical conductivity can be extracted from fits to the data given the sphere radius only. The same apparatus is also used to explore the effects of geometry on eddy current generation as well as to demonstrate non-destructive testing through measurements on coins of different composition. (c) 2018 American Association of Physics Teachers. https://doi.org/10.1119/1.5034350
\end{abstract}

\section{INTRODUCTION}

Exactly solvable electromagnetic problems-which provide important models for pedagogy as well as researchgenerally possess planar, cylindrical, or spherical symmetry. The standard textbook presentation of the electromagnetic skin depth, for example, stems from the solution to the problem of an electromagnetic plane wave incident on the surface of a conducting medium that completely fills half-space. ${ }^{1,2}$ The main result of this analysis-namely, the exponential attenuation of electromagnetic fields within the bulk of a conductor-provides valuable insight into the general problem of eddy currents and remains valid for any geometry as long as the skin depth is small compared to the curvature of the conductor. Exact solutions for specific geometries such as conducting slabs, cylinders, and spheres are known and can be found in textbooks ${ }^{3-5}$ as well as throughout the past $^{6-8}$ and more recent literature. ${ }^{9-14}$

Of these geometries, a sphere in a uniform ac field is of particular interest in that it represents an exactly solvable model for a finite-sized object. As a result, it can be represented faithfully in a simple experiment without any approximation as will be shown here. This alone has great pedagogical value. And despite the naivety of the geometry, the sphere in a uniform ac field continues to be a valuable model in research. Some recent examples include the following: the absorption of electromagnetic power in biological tissue $^{12,15}$ and metallic implants ${ }^{16}$ during the magnetic resonance imaging process; the industrial inspection of agricultural produce; ${ }^{17}$ and the de-spinning of metallic space objects. ${ }^{18}$ Generalization to the prolate spheroid ${ }^{19}$ extends the utility of this model.

Another very important field of research is eddy-currentbased non-destructive testing, which has many widespread applications such as the detection of buried munitions, ${ }^{20}$ the detection of defects in aircraft structures, ${ }^{21}$ and the inspection of railroad tracks. ${ }^{22}$ These techniques employ aspects of electromagnetic theory that are readily accessible to the undergraduate student. The study and experimental verification of the response of a metallic sphere in a uniform ac field provides an excellent introduction to nondestructive testing as well as the general concepts of eddy current behavior. $^{5,23-28}$
The theory and experiment presented here are highly suitable for an undergraduate laboratory. Through careful design of the experimental procedure, high-quality data can be collected with relative ease. Data are fit to the theoretical model of the magnetic moment of the metallic sphere, from which precise values of the magnetic permeability $\mu$ and electrical conductivity $\sigma$ are extracted. A qualitative component of the experiment allows students to explore aspects of nondestructive testing with samples of different shape and composition. The simplicity, precision, and flexibility of our apparatus provide advantages over similar experiments proposed for undergraduate teaching. ${ }^{26-28}$

This paper is organized as follows. First we review the theory of a metallic sphere in a uniform ac field, focusing on the induced magnetic moment and the accompanying dipole field, which can be measured directly. Next we describe the apparatus and the experimental procedure developed to obtain high-precision data. We then present and discuss results for a variety of non-magnetic and magnetic metal spheres. Lastly, we describe two ancillary experiments which rely on the qualitative comparison of data to demonstrate other applications of eddy-current-based non-destructive testing methods.

\section{THEORY}

We consider a sphere made of a linear medium with radius $a$, permittivity $\epsilon$, permeability $\mu$, and conductivity $\sigma$ in an oscillating, uniform applied field $\boldsymbol{B}(t)=B e^{-i \omega t} \hat{\boldsymbol{z}}$ of frequency $f=\omega / 2 \pi$. From Lenz's Law and the right-hand-rule, one expects the rise of induced currents (i.e., eddy currents) flowing in the azimuthal direction to oppose the change of flux in the sphere. The eddy current density has a $\sin \theta$ distribution with regard to the polar angle, ${ }^{12}$ and is zero at the poles and maximal at the equator as depicted in Fig. 1. The flow of current in the sphere gives rise in turn to a magnetic moment $\boldsymbol{m}=m \hat{\boldsymbol{z}}$ of complex amplitude

$$
m=\frac{2 \pi a^{3} B}{\mu_{0}} \frac{2\left(\mu_{r}-1\right) j_{0}(k a)+\left(2 \mu_{r}+1\right) j_{2}(k a)}{\left(\mu_{r}+2\right) j_{0}(k a)+\left(\mu_{r}-1\right) j_{2}(k a)},
$$

where the explicit time dependence has been dropped for simplicity, $j_{0}$ and $j_{2}$ are the zeroth and second-order spherical 
Bessel functions, $k=\sqrt{\mu \epsilon \omega^{2}+i \mu \sigma \omega}$ is the propagation constant with $i=\sqrt{-1}$, and $\mu_{r}=\mu / \mu_{0}$ is the relative permeability. The derivation of this result and the magnetic field generated by the sphere ${ }^{12}$ should be accessible to any student having read an undergraduate textbook on electromagnetism. ${ }^{1,5,23}$ For a non-magnetic sphere $\left(\mu_{r}=1\right)$, Eq. (1) becomes ${ }^{4,5}$

$$
m=-\frac{2 \pi a^{3} B}{\mu_{0}}\left(1+\frac{3}{k a} \cot (k a)-\frac{3}{(k a)^{2}}\right) .
$$

In the region outside the sphere $(r>a)$, the components of the net magnetic field are

$$
\begin{aligned}
& B_{r}=\left(B+\frac{2 \mu_{0} m}{4 \pi r^{3}}\right) \cos \theta, \\
& B_{\theta}=\left(-B+\frac{\mu_{0} m}{4 \pi r^{3}}\right) \sin \theta,
\end{aligned}
$$

which is the superposition of the applied field $\boldsymbol{B}$ and the induced dipole field from the sphere. Converting to cylindrical coordinates, with $r^{2}=\rho^{2}+z^{2}$, gives

$$
\begin{aligned}
& B_{\rho}=\frac{3 \mu_{0} m}{4 \pi} \frac{\rho z}{r^{5}} \\
& B_{z}=B+\frac{\mu_{0} m}{4 \pi} \frac{2 z^{2}-\rho^{2}}{r^{5}},
\end{aligned}
$$

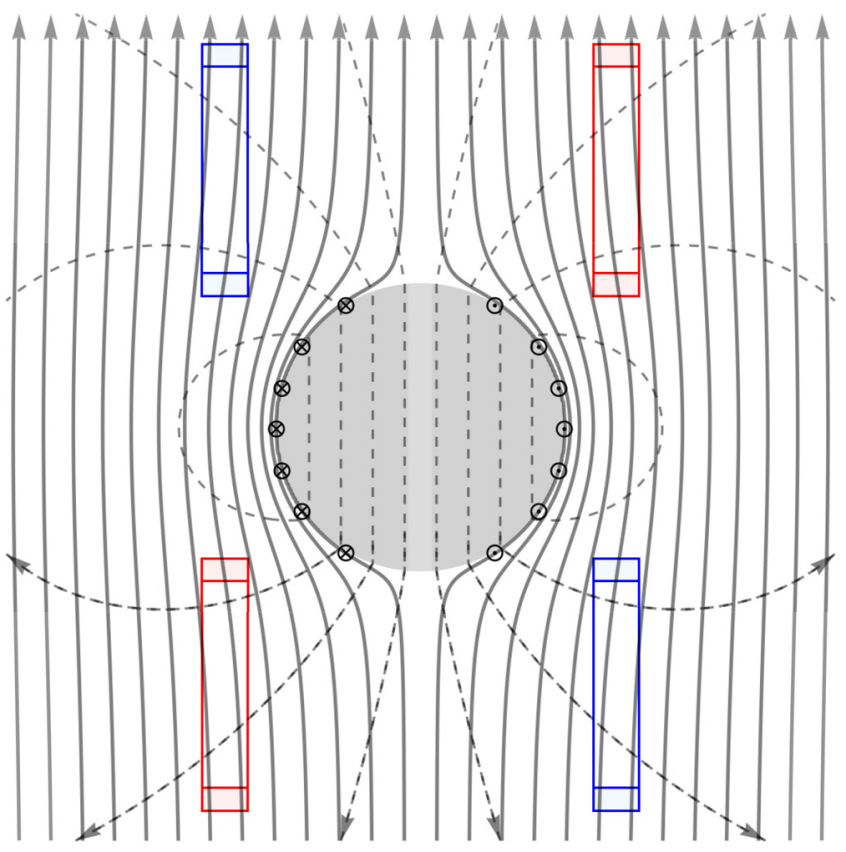

Fig. 1. Conceptual diagram of the experimental method showing the metallic sphere in the thick limit. The solid lines are the net magnetic field (which is zero inside the sphere when $a \gg \delta$ ), while the dashed lines are the contribution from just the induced magnetic moment of the sphere. The distribution of eddy current flow in the azimuthal direction is indicated by the dots and crosses on the surface of the sphere. Identical pick-up coils with the plane of their windings aligned along the applied field are sensitive only to the field due to the sphere, with either positive $(+)$ or negative $(-)$ flux as distinguished by the direction of the field lines passing through them. A differential measurement of the induced emfs (e.g., $\mathcal{E}_{+}-\mathcal{E}_{-}$) doubles the sample signal and nullifies noise from background fields. from which one sees that $B_{\rho}$ is due solely to the magnetic moment of the sphere. This offers an opportunity, then, to determine $m$ through a direct measurement of the dipole field that is unobscured by the applied field.

If one considers inductive detection of $B_{\rho}$, then the flux through a pick-up coil (PC) that has the plane of its windings aligned along the applied field as shown in Fig. 1 is

$$
\Phi=\int_{\mathrm{PC}} B_{\rho} \mathrm{d} s=\frac{3 \mu_{0} m}{4 \pi} \int_{\mathrm{PC}} \frac{\rho z}{r^{5}} \mathrm{~d} s,
$$

where $\mathrm{d} s$ is the surface element of the coil. The integral on the right hand side of Eq. (7) is a geometric factor that depends only on the turns, dimensions, and relative location of the coil. Once these are fixed, the emf $\mathcal{E}$ induced in the coil is simply a constant multiplied by $\omega m$. As a result, it is possible given the sphere radius $a$ to extract values of $\mu, \epsilon$, and $\sigma$ by fitting measurement data of $\mathcal{E}(\omega) / \omega$ to Eq. (1). This is the approach used here, and it will be described further in Sec. III.

Before proceeding, we first discuss the specific theoretical behaviour of the metallic sphere pertinent to this work. In particular, we note that the quasistatic limit $\omega \epsilon \ll \sigma$, which is generally applicable for good conductors, ${ }^{1}$ will be very well satisfied over the entire frequency range explored here. As a result, the propagation constant in Eq. (1) safely reduces to $k=\sqrt{i \mu \sigma \omega}$. The expansion of Eq. (1) in the limit $\omega \rightarrow 0$ gives

$$
\begin{aligned}
& \operatorname{Re}\{m\}=\frac{4 \pi a^{3} B}{\mu_{0}}\left[\frac{\mu_{r}-1}{\mu_{r}+2}-\frac{6\left(\mu_{r}^{2}+9 \mu_{r}\right) a^{4}}{175\left(\mu_{r}+2\right)^{3} \delta^{4}}\right], \\
& \operatorname{Im}\{m\}=\frac{4 \pi a^{3} B}{\mu_{0}}\left[\frac{3 \mu_{r} a^{2}}{5\left(\mu_{r}+2\right)^{2} \delta^{2}}\right],
\end{aligned}
$$

where $\operatorname{Re}\{m\}$ and $\operatorname{Im}\{m\}$ are the real and imaginary parts of $m$, respectively, and $\delta=\sqrt{2 / \mu_{r} \mu_{0} \sigma \omega}$ is the electromagnetic skin depth for metals in the quasistatic limit.

Figure 2 shows theoretical plots of $m(f)$ for typical parameters of the metallic spheres used in this work. The dc limit of Eq. (8) is evident here: $m$ is zero for non-magnetic metals (i.e., $\mu_{r}=1$ ) but remains finite for magnetic metals (e.g., $\left.\mu_{r}>1\right)$. As the frequency increases, eddy-current shielding eventually overtakes bulk magnetization and the real component of $m$, which is in-phase with $\boldsymbol{B}(t)$, changes sign and reaches an asymptotic value, indicating the complete expulsion of flux from inside the sphere. This is the thick limit (i.e., $a \gg \delta$ ) depicted in Fig. 1.

It is also well known that in this limit the induced eddycurrent density oscillates spatially within the exponential decay envelope set by the skin depth. ${ }^{1-5}$ As $\delta \rightarrow 0$, the counterflowing contributions of the out-of-phase component of the current density produce zero net field outside the sphere. As a result, the imaginary component of $m$, which initially increases with frequency as a simple consequence of Faraday's Law, goes to zero as $f \rightarrow \infty$ as can also be seen in Fig. 2 .

At this point, it is worth emphasizing that the above analysis-though of considerable practical and pedagogical value-is generally incomplete in that it assumes all material properties to be field and frequency independent. One does expect, however, that both the permittivity and conductivity will be constant at the very low frequencies examined here, 


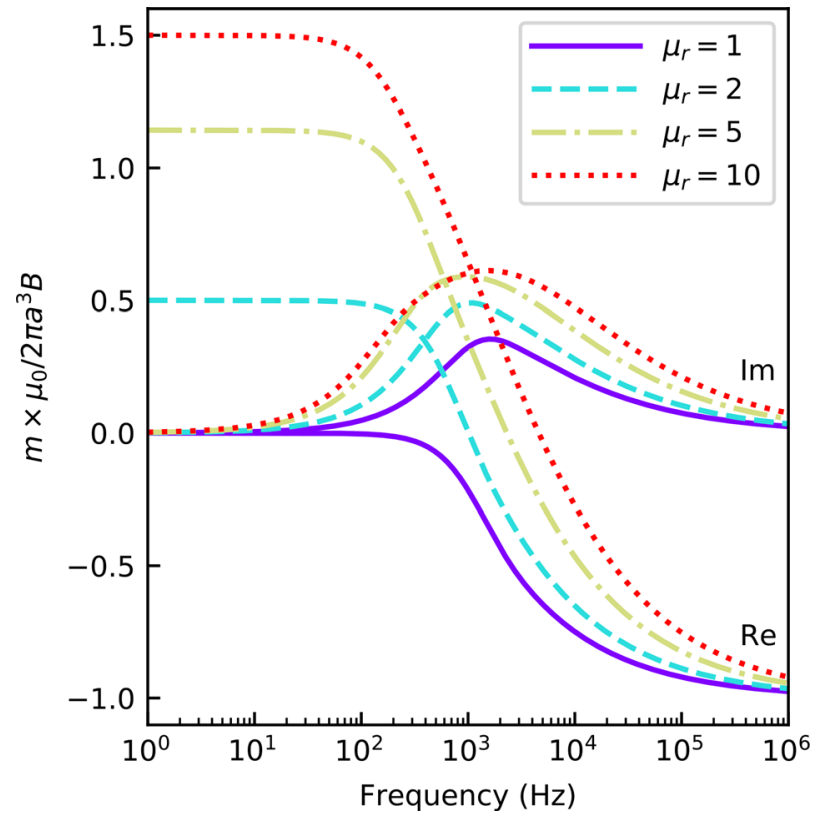

Fig. 2. Theoretical plots of Eq. (1) with $k=\sqrt{i \mu_{0} \mu_{r} \sigma \omega}$ for spheres of diameter $3 / 8^{\prime \prime}$, electrical conductivity $10^{7} \mathrm{~S} / \mathrm{m}$, and various values of $\mu_{r}$. The real (Re) and imaginary $(\mathrm{Im})$ components of $m$ are in-phase and out-of-phase with the applied field $\boldsymbol{B}(t)$, respectively.

which can be seen from even fairly simple models of electron motion. ${ }^{1,2,5,29}$ The permeability, on the other hand, deserves a closer look.

Excellent discussions on the various forms of magnetism in matter can be found in several textbooks at the introductory ${ }^{5,23,30}$ and more advanced ${ }^{29}$ level. Following common parlance, we consider here nominally non-magnetic (i.e., diamagnetic or paramagnetic) and magnetic (i.e., ferromagnetic) materials. The former arise, respectively, from the response of the electronic orbital and spin magnetic moments to an applied magnetic field. These effects are generally quite weak and can be well characterized by a constant magnetic susceptibility $\left(\chi_{m}=\mu_{r}-1\right)$ that is negative for diamagnets and positive for paramagnets, and which is of order $10^{-5}-10^{-4}$ for typical metals. ${ }^{1}$ Ferromagnets, on the other hand, are inherently nonlinear materials that are characterized by the spontaneous and near complete alignment of unpaired electron spins within small magnetic domains. Ferromagnets also typically exhibit hysteresis, and the application of an external magnetic field can force changes in the orientation and boundaries of the domains that result, for example, in the permanent magnetization, saturation, or possibly demagnetization of a sample depending on its history and the manner in which the field is applied. In light of its nonlinearity and domain dynamics, one expects the general ferromagnet to have a permeability that is both field and frequency dependent. While such effects will not be explicitly incorporated into the aforementioned model for a magnetic metallic sphere, we do show below how $\mu_{r}(f)$ can be extracted from the data for such a sample.

\section{EXPERIMENTAL METHOD}

The apparatus is shown in Fig. 3 and comprises a Helmholtz coil to generate the uniform applied field $\boldsymbol{B}(t)$ and a pair of identical coils harvested from a defunct computer hard drive to serve as pick-up coils. The Helmholtz coil has
20 turns per loop, with a total resistance of $1.2 \Omega$, an inductance of $0.56 \mathrm{mH}$, and an efficiency of $150 \mu \mathrm{T} / \mathrm{A}$. Each pickup coil has 77 turns, a resistance of $7.3 \Omega$, and an inductance of $8.2 \mathrm{mH}$. One could use four pick-up coils (as shown in Fig. 1) to increase sample signal, but for ease of construction and for greater access to the sample space, we chose to use only two. It is important, however, that pairs of pick-up coils be as close to identical as possible in order to effectively cancel spurious signal from any background fields.

A lock-in amplifier (Stanford Research SR830) under computer control is used to drive the Helmholtz coil and measure the differential signal (A-B mode) between the two pick-up coils. The following settings of the lock-in were fixed for all experiments: sine output $=5 \mathrm{~V}_{\text {rms }}$, reference phase $=0^{\circ}$, and time constant $=300 \mathrm{~ms}$. In-house software is used to change the lock-in frequency in logarithmically spaced steps over the range of study (from $25 \mathrm{~Hz}$ to the lockin limit of $102 \mathrm{kHz}$ ), to automatically adjust the lock-in sensitivity so that signal is always between half- and full-scale, and to record the demodulated signal of the two lock-in output channels (X and Y). To ensure independent data points free from any transient effects, the program waits more than five time constants after any changes before monitoring $\mathrm{X}$ and $\mathrm{Y}$.

In order to accurately determine $\mu_{r}$ and $\sigma$ for the metallic sphere, three sets of measurements are needed: (1) a normalization run to determine the magnitude and phase of the applied field $\boldsymbol{B}(t)$, which are frequency dependent due to the complex impedance of the drive circuit, (2) a background run performed without the sample present to determine any frequency-dependent coupling between the drive and pickup coils due to imperfect alignment, and (3) a foreground run with the sample present. The set-up and typical results of each type of measurement are shown in Fig. 4. As described further below, one subtracts the background from the foreground, then phases and normalizes this result before fitting to the model. The normalization and background runs need

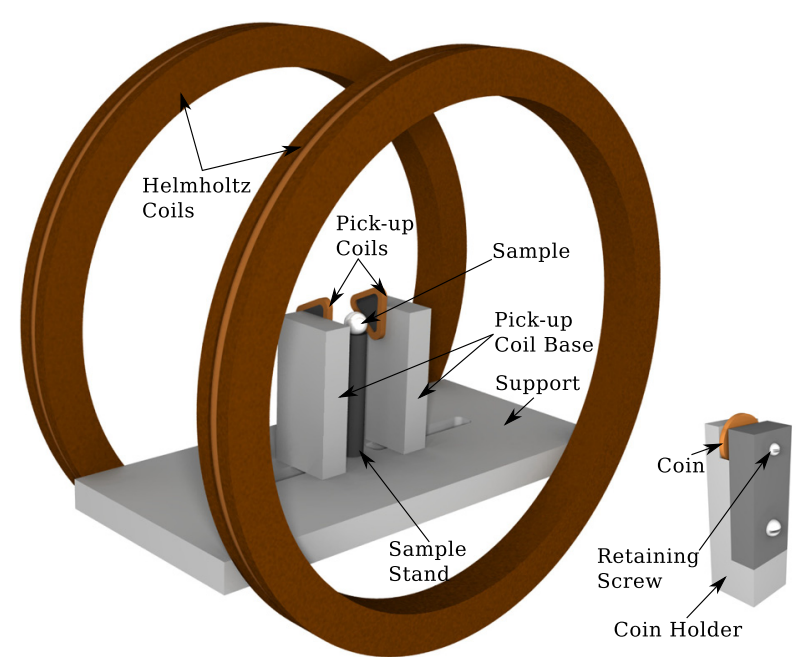

Fig. 3. Model of the apparatus. The Helmholtz coil generates the uniform field $\boldsymbol{B}(t)$. As displayed here, the pick-up coils are positioned about $24 \mathrm{~mm}$ apart with their one edge aligned with the center of a metallic sphere of diameter $3 / 8^{\prime \prime} \simeq 9.5 \mathrm{~mm}$. The pick-up coil spacing can be adjusted to accommodate samples of different sizes. The top of the sample stand is bored out to accurately position the metallic spheres. In later experiments, the stand is replaced with a coin holder which features a retaining screw to ensure that coins are positioned identically across multiple trials. 
be performed only once and can be used across multiple foreground runs, including those with different samples.

The emf of the normalization run at any given frequency is

$$
\mathcal{E}_{\text {norm }}=-i \omega \tilde{B} \int_{\mathrm{PCs}} \mathrm{d} s_{z},
$$

where $\tilde{B}=B e^{i \phi_{B}}$ and $\mathrm{d} s_{z}$ is the projection of the surface element $\mathbf{d} \boldsymbol{s}$ of the pick-up coils along the $z$-direction parallel to $\boldsymbol{B}$. The phase of the applied field is determined at each frequency from the lock-in outputs as $\phi_{B}=\arctan \left(\mathrm{Y}_{\text {norm }} / \mathrm{X}_{\text {norm }}\right)$.

The net emf due to the sample is the difference between the foreground and background runs and is determined at each frequency as $\mathcal{E}_{\text {samp }}=\mathcal{E}_{\text {fore }}-\mathcal{E}_{\text {back }}$. To account for the phase of the applied field, and to allow for a proper fit to the data, one must rotate $\mathcal{E}_{\text {samp }}$ to $\mathcal{E}_{\text {samp }}^{\prime}$ in the complex plane by the following operation:

$$
\left(\begin{array}{c}
\mathrm{X}_{\mathrm{samp}}^{\prime} \\
\mathrm{Y}_{\mathrm{samp}}^{\prime}
\end{array}\right)=\left(\begin{array}{cc}
-\cos \left(\phi_{B}\right) & -\sin \left(\phi_{B}\right) \\
-\sin \left(\phi_{B}\right) & +\cos \left(\phi_{B}\right)
\end{array}\right)\left(\begin{array}{c}
\mathrm{X}_{\mathrm{samp}} \\
\mathrm{Y}_{\mathrm{samp}}
\end{array}\right) .
$$

One can now associate the in-phase $\left(\mathrm{X}_{\text {samp }}^{\prime}\right)$ and out-of-phase ( $\mathrm{Y}_{\text {samp }}^{\prime}$ ) components of $\mathcal{E}_{\text {samp }}^{\prime}$ with those of the magnetic moment $m$.

Making use of Eqs. (1), (7), and (10), a final fit function is determined:

$$
\frac{\mathcal{E}_{\text {samp }}^{\prime}}{\left|\mathcal{E}_{\text {norm }}\right|}=a^{3} G \frac{2\left(\mu_{r}-1\right) j_{0}(k a)+\left(2 \mu_{r}+1\right) j_{2}(k a)}{\left(\mu_{r}+2\right) j_{0}(k a)+\left(\mu_{r}-1\right) j_{2}(k a)},
$$

where the constant

$$
G=3 \int_{\mathrm{PCs}} \frac{\rho z}{r^{5}} \mathrm{~d} s \div 2 \int_{\mathrm{PCs}} \mathrm{d} s_{z}
$$

is a purely geometric scaling factor associated with the pickup coils that is independent of the size and material properties of the spherical sample. The normalization process eliminates the factor $\omega B$ arising from Faraday's Law, as well as any other frequency dependence that may be present in the gain of the receive chain. By including $G$ as a third free parameter in the fitting routine (along with $\mu_{r}$ and $\sigma$ ), explicit knowledge of pick-up coil dimensions and locations is not needed. A least-squares fitting algorithm from Python's SciPy package is used to simultaneously fit the real and imaginary parts of Eq. (12) to the data after all post processing steps are complete. For a non-magnetic sphere $\left(\mu_{r}=1\right)$, the fit function becomes

$$
\frac{\mathcal{E}_{\text {samp }}^{\prime}}{\left|\mathcal{E}_{\text {norm }}\right|}=-a^{3} G\left(1+\frac{3}{k a} \cot (k a)-\frac{3}{(k a)^{2}}\right),
$$

with only two free parameters $G$ and $\sigma$.

\section{RESULTS AND DISCUSSION}

\section{A. Quantitative experiments with metallic spheres}

The measurement procedure described above was performed using metallic spheres of nominal diameter $3 / 8^{\prime \prime}$ that were purchased commercially. Four different materials were tested-aluminum (Type 2017-T4), brass, tungsten carbide, and stainless steel (Type 440c) - giving a variety of nonmagnetic and magnetic samples.

The final phased and normalized data, along with fits to theory and residuals, are shown in Figs. 5 and 6. As can be seen, the quality of the data produced by this very simple apparatus and experimental method is very high. The signalto-noise ratio of the experiment degrades quickly below $25 \mathrm{~Hz}$, however, and data were not collected in this region. The data fit very well with theory over the entire frequency range for all samples, with stainless steel showing the most prominent deviations. This may be due to a frequencydependent permeability, which is not included in the present model and will be discussed further below.

A summary of all quantitative results is given in Table I, along with reference values for $\mu_{r}$ and $\sigma$ that could be found in the literature. ${ }^{31-35}$ The results are wholly consistent with the qualitative behavior of the response curves in Figs. 5 and 6.
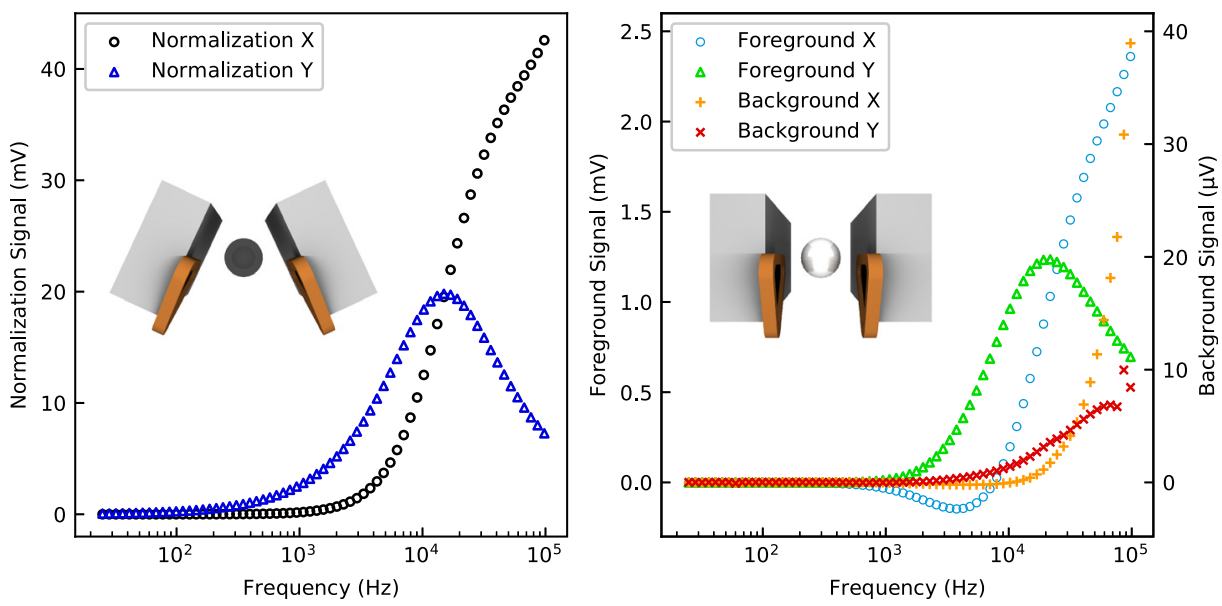

Fig. 4. The experimental set-up and results for a typical normalization measurement (left) and foreground and background measurements (right). The normalization measurement is made with the pick-up coils turned toward the applied field. The choice of angle is not critical and does not need to be known, but should be sufficient to provide an accurate measure of $B$. The frequency dependence of the normalization signal is dominated by the $50 \Omega$ output of the lock-in amplifier in series with the reactance of the Helmholtz coil. Alignment of the pick-up coils along $\boldsymbol{B}$ (right) is achieved by minimizing the background signal at a fixed frequency $(10 \mathrm{kHz}$, say) through manual adjustment of each coil separately. Since perfect decoupling can never be achieved, a final background measurement versus frequency is needed. The frequency dependence of the foreground signal is dominated by the response of the metallic sphere, which in turn is proportional to $B$. As a result, the gross features of the foreground and normalization signals are similar. 

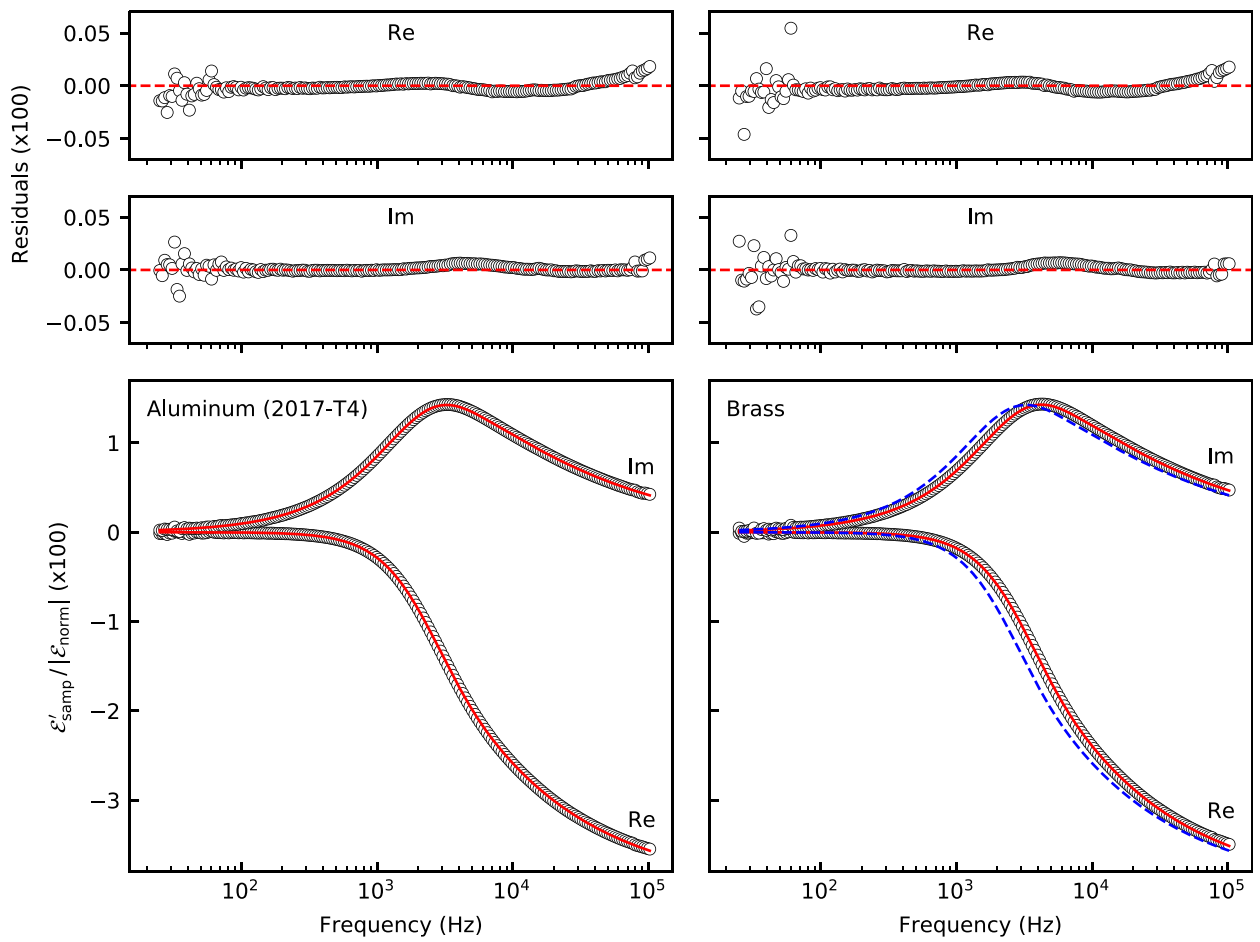

Fig. 5. Response of the aluminum and brass spheres: data (circles), fit function for the non-magnetic model (solid lines). Corresponding fits to the magnetic model (see Table I) are indistinguishable by eye and not shown for clarity. For ease of comparison, the fit function for aluminum is superimposed on the graph for brass (dashed lines). Top panels: Residuals (circles) with zero highlighted for clarity (dashed lines).

Aluminum and brass are non-magnetic, with both exhibiting zero signal as $f \rightarrow 0$. Of the two, aluminum has the larger electrical conductivity, as seen by the lower frequency peak in the out-of-phase response and the more negative in-phase response over the entire frequency range. The tungsten carbide and stainless steel samples, on the other hand, are clearly magnetic as indicated by the large positive $y$-intercept of their in-phase response. (Ferromagnetic impurities, such as nickel or cobalt, are likely responsible for the magnetism of the former. ${ }^{34}$ ) Of the two samples, stainless steel has the larger permeability; it also has the lower electrical conductivity. Both a larger $\mu_{r}$ and a lower $\sigma$ push the asymptotic limits of the in- and out-of-phase response to higher frequency as compared to the tungsten carbide sample.

The large number of data taken for each sample leads to the high statistical precision of the fit parameters seen in Table I. Such precision should be interpreted with caution, however, as it exceeds the true capabilities of the apparatus. This can be seen from two particular aspects of the results. First, the values of the scaling factor $G$, which should be equivalent for all samples here since the same normalization run was used for each, do not agree at the level of their respective uncertainties. Taking the mean and standard deviation of all $G$ values (excluding stainless steel, which has the poorest fit to the model) gives $G=(3.74 \pm 0.01) \times 10^{5} \mathrm{~m}^{-3}$. This suggests that the reproducibility or measurement precision of our method is at the level of three parts per thousand, which is around an order of magnitude larger than typical fit parameter uncertainties. Second, the fits for aluminum and brass to the magnetic model would suggest that both materials are diamagnetic $\left(\mu_{r}<1\right)$. Not only is the sign of the magnetic susceptibility $\chi_{m}$ wrong for aluminum, its magnitude is roughly $40 \times$ larger than the expected paramagnetic value. Furthermore, the uncertainty in the fit value is about

Table I. Measured sphere diameters; fit parameters for the data in Figs. 5 and 6; and reference values for $\mu_{r}$ and $\sigma$. Electrical conductivities are given in units of mega-Siemens per meter. The numbers in parentheses are the uncertainty in the last digit of the measured and fit quantities. The second rows for aluminum and brass are fits to the non-magnetic model, which assumes $\mu_{r}$ to be unity. The second row for stainless steel is a two-parameter fit to the magnetic model assuming a known value of $G=3.74 \times 10^{5} \mathrm{~m}^{-3}$ as discussed in the text.

\begin{tabular}{|c|c|c|c|c|c|c|}
\hline \multirow[b]{2}{*}{ Sphere material } & \multirow{2}{*}{$\begin{array}{l}\text { Diameter } \\
2 a(\mathrm{~mm})\end{array}$} & \multicolumn{3}{|c|}{ Fit parameters } & \multicolumn{2}{|c|}{ Reference values [31-35] } \\
\hline & & $\mathrm{G}\left(10^{5} \mathrm{~m}^{-3}\right)$ & $\mu_{r}$ & $\sigma(\mathrm{MS} / \mathrm{m})$ & $\mu_{r}$ & $\sigma(\mathrm{MS} / \mathrm{m})$ \\
\hline Aluminium (2017-T4) [31,32] & $9.500(5)$ & $\begin{array}{l}3.743(1) \\
3.743(1)\end{array}$ & $\begin{array}{c}0.9991(3) \\
1\end{array}$ & $\begin{array}{l}20.03(2) \\
20.04(2)\end{array}$ & 1.000021 & 20 \\
\hline Brass [33] & $9.500(5)$ & $\begin{array}{l}3.753(2) \\
3.752(2)\end{array}$ & $\begin{array}{c}0.9990(3) \\
1\end{array}$ & $\begin{array}{l}15.43(2) \\
15.45(2)\end{array}$ & 1.0 & 15 \\
\hline Tungsten carbide [34] & $9.510(5)$ & $3.732(2)$ & $1.8359(8)$ & $5.436(5)$ & - & 5 \\
\hline Stainless steel (440c) [35] & $9.510(5)$ & $\begin{array}{c}3.776(2) \\
3.74\end{array}$ & $\begin{array}{c}15.97(7) \\
16.7(1)\end{array}$ & $\begin{array}{c}1.473(4) \\
1.51(1)\end{array}$ & - & 1.7 \\
\hline
\end{tabular}



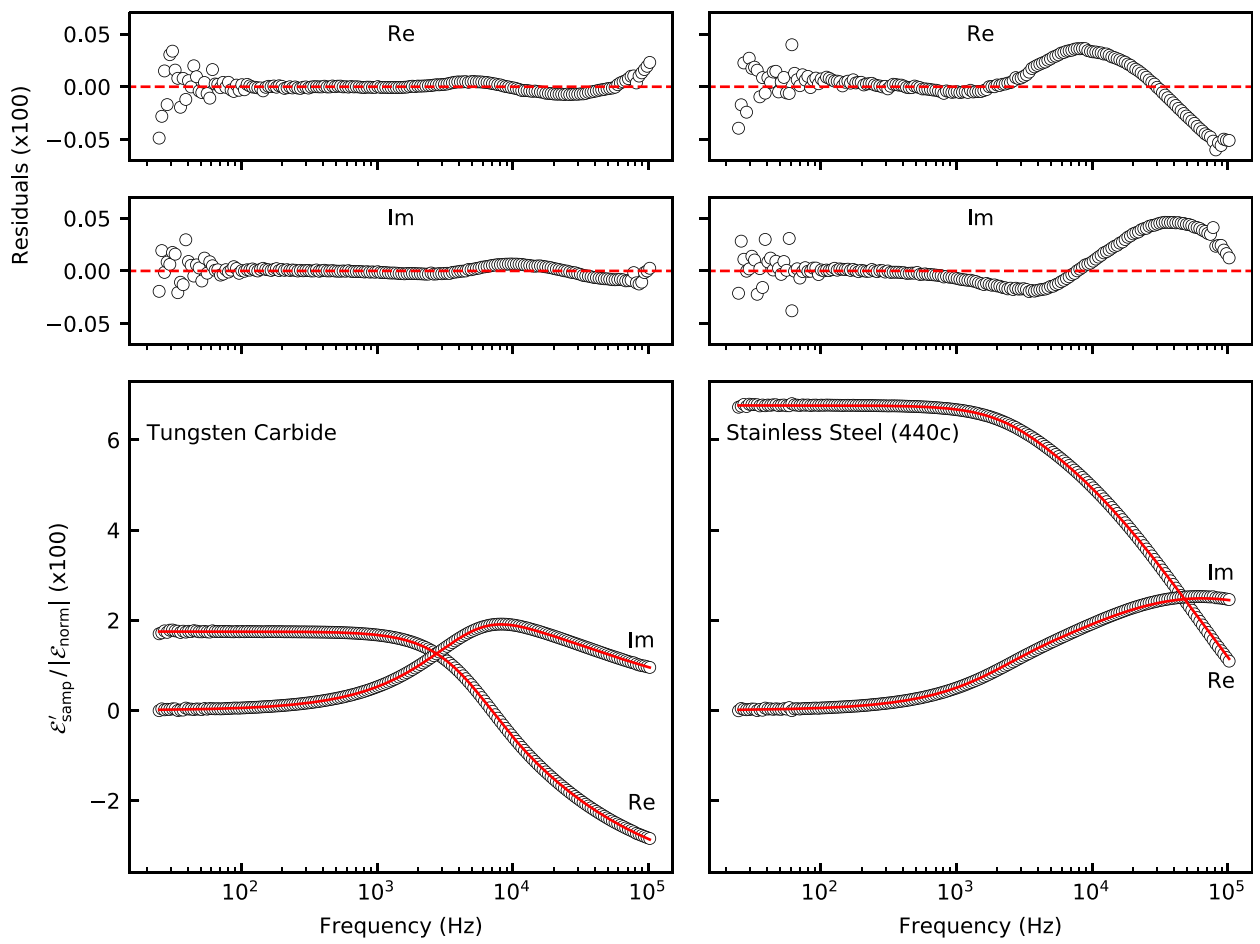

Fig. 6. Response of the tungsten carbide and stainless steel spheres: data (circles), fit function for the magnetic model (solid lines). The two- and threeparameter fits made for stainless steel (see Table I) are marginally distinguishable by eye and only the former is shown here for clarity. Top panels: Residuals (circles) with zero highlighted for clarity (dashed lines).

$15 \times$ larger than the expected susceptibility. The conclusion is that this apparatus does not have the resolution to measure the weak susceptibilities of diamagnetic and paramagnetic materials, and that fits to the non-magnetic model are sufficient in this case. This in turn suggests an accuracy of no better than one part per thousand in measurements of permeability in order for the results of aluminum and brass to be consistent with $\mu_{r}=1$.

To further explore the limitations of this measurement technique, we ran four successive trials with the aluminum sphere, each time repeating the normalization run, the background run, and the foreground run. The data were fit to the magnetic model. It is not expected that $G$ should be the same for all trials here, as it depends on the precise location of the coils from run to run. The mean and standard deviation of the other fit parameters over the four trials were found to be $\mu_{r}=1.0003 \pm 0.0009$ and $\sigma=19.83 \pm 0.06 \mathrm{MS} / \mathrm{m}$. Again, one concludes that $\chi_{m}$ cannot be resolved for such weakly magnetic materials, and that a reasonable estimate for the accuracy on $\mu_{r}$ is one part per thousand. The results for the electrical conductivity suggest a measurement precision of three parts per thousand. The disagreement between the value of $\sigma$ determined here and that reported in Table I is roughly $1 \%$, from which a reasonable estimate for the accuracy on $\sigma$ is five parts per thousand.

With a better understanding of the capabilities of our apparatus, we now comment on the reference values for $\sigma$ given in Table I. There is generally good agreement with our measurements, especially for aluminum and brass, although the uncertainty in the reference values (if one assumes half of the last digit) is at least $3 \%$ in all cases. This may suggest that the variability in the electrical conductivities of nominally identical materials (such as Type 2017-T4 aluminum) is typically an order of magnitude larger than the precision and accuracy of our measurements. This could be tested in the future with multiple spherical samples of the same nominal material made from different stock. This same observation also highlights the potential of our simple apparatus and method for characterizing conductive materials.

Finally, we discuss in greater detail the results for the two magnetic materials. In particular, we note that tungsten carbide, given its similar fit value of $G$ and magnitude of residuals compared to brass and aluminum, is well described by a constant $\mu_{r}$ and $\sigma$ over the range of frequencies explored here. Stainless steel on the other hand is not. First of all, the value of $G$ from its three-parameter fit deviates notably from the other samples. Since $G$ is a geometric factor associated with the shape and position of the pickup coils, it should not depend on the sample of course. The fact that it does is an indication the present model with constant $\mu_{r}$ and $\sigma$ is insufficient.

To explore this further, we first reanalyzed the data for stainless steel using the average value for $G$ from the other samples as input to the model. This is akin to calibrating the apparatus using a known standard. The resulting twoparameter fit returns slightly different constant values of $\mu_{r}$ and $\sigma$ (see Table I), but the residuals (see Fig. 6) did not show any marked improvement. Assuming that the conductivity of stainless steel is frequency independent, as argued above, we subsequently extracted $\mu_{r}$ from Eq. (12) at each frequency with $G=3.74 \times 10^{5} \mathrm{~m}^{-3}$ and $\sigma=15.5 \mathrm{MS} / \mathrm{m}$. This estimate for the dc value of $\sigma$ came from a twoparameter fit over the low frequency range of the data up to $500 \mathrm{~Hz}$. The resulting data for the putative $\mu_{r}(f)$ of the stainless steel sample are presented in Fig. 7 and show a clear resonance-like variation of about $8 \%$. This could be a signature of magnetic relaxation due to the phase lag of domainwall motion with respect to the applied field. ${ }^{36}$ Further 
studies, which are beyond the scope of this present work, would require a complex model of the permeability as well as careful preparation of the magnetic history of the sample. ${ }^{36}$ Such studies could also benefit from smaller sample sizes (to minimize eddy-current effects) as well as an independent determination of dc conductivity. Still, the initial results presented here demonstrate the potential of this method for exploring the physics associated with strongly magnetic materials, such as hysteresis, ordering, and domain-wall dynamics.

\section{B. Qualitative experiments and non-destructive testing}

The accurate determination of electromagnetic properties requires a well-defined sample shape and accompanying model, as demonstrated in Sec. IV A. Eddy-current-based non-destructive testing does not necessarily require an explicit determination of $\mu_{r}$ and $\sigma$, however, and the qualitative analysis of metallic samples-either by comparison against a standard or by comparison between samples — still offers valuable information. Two additional experiments involving non-spherical samples are presented here that demonstrate both methods of comparison and highlight some of the principles and uses of non-destructive testing.

\section{Orientation of a non-spherical sample}

In this experiment, we compare the response of an intact brass sphere to one that has had roughly $0.9 \mathrm{~mm}$ of material removed from opposite sides using a file. The former sample can be thought of as a standard, while the latter represents a test piece that has been compromised by wear or deformation, say. Using the same procedure and sample holder as above, measurements were made with the applied field $\boldsymbol{B}(t)$ either parallel or perpendicular to the normal of the flat face of the test piece as shown in Fig. 8. A general discussion regarding the orientation of an ac magnetic field to a metal surface can be found in Ref. 5 .

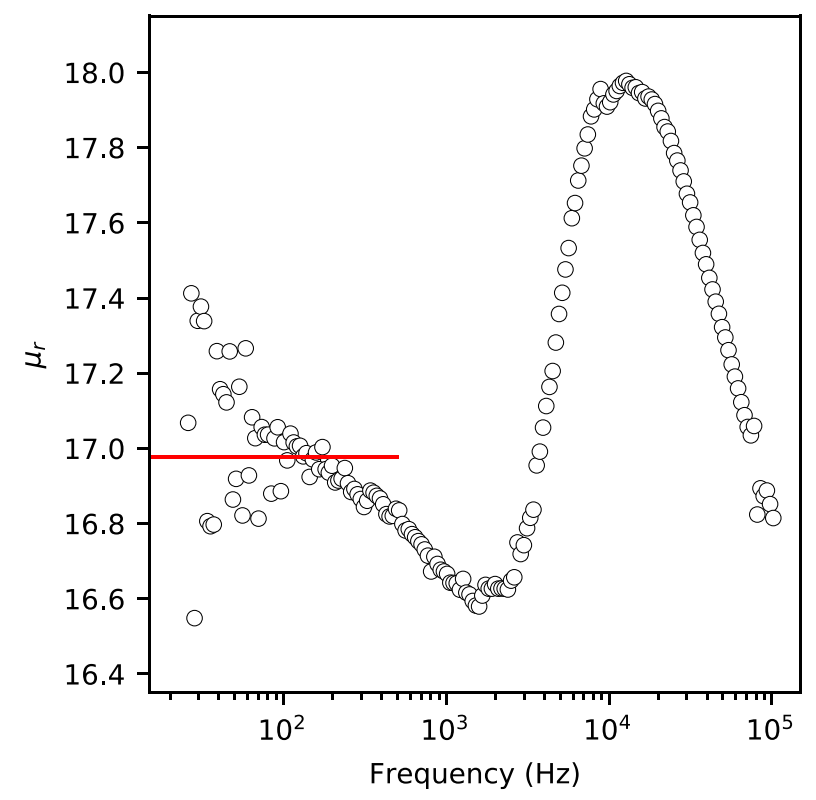

Fig. 7. A point-by-point extraction of the relative permeability of the stainless steel sphere from Eq. (12) assuming a known $G$ and $\sigma$. The horizontal line is the constant value of $\mu_{r}$ from the two-parameter fit of the low frequency data used to determine $\sigma$ as discussed in the text.
The results are shown in Fig. 8, and one can see that while the parallel case is almost indistinguishable from the intact sphere, the perpendicular case is notably different. These results highlight an important aspect of non-destructive testing, namely, that there must be a sufficient perturbation or disruption of the eddy current flow pattern compared to that of the standard in order to identity the existence of damage or deformation in a test piece.

In regard to the particular case studied here, it is clear that the eddy current density of the intact sphere must be zero along the axis of the applied filed, as given by the $\sin \theta$ dependence of $J_{\phi}$ in Eq. (8) of Ref. 12. As a result, removing material near the poles of the sphere (i.e., the parallel case) has little effect on the bulk of the eddy current flow which is primarily near the equator. In contrast, removing material near the equator (i.e., the perpendicular case) strongly alters the path of the eddy currents and in turn modifies the magnitude and spatial distribution of the associated magnetic field.

\section{Coins of different composition}

In this experiment, we compare the response of Canadian 1-cent coins that are more or less identical in shape, but whose metallic composition depends on year of minting. ${ }^{37}$ Prior to 1997, the composition was $98 \%$ copper, $1.75 \%$ tin and $0.25 \%$ zinc; for the years 1997 to 1999 , the composition was $98.4 \%$ zinc with $1.6 \%$ copper as plating; from 2000 onward, a second composition was introduced that comprises $94 \%$ steel and $1.5 \%$ nickel with $4.5 \%$ copper as plating. Four coins were tested: one from 1996, one from 1999, and two from 2003. Visually, all appear to be identical copper coins. Measurements are made with $\boldsymbol{B}(t)$ applied normal to the coin face. The coins are held in a well-defined position relative to the drive and pick-up coils using the coin holder shown in Fig. 3. This allows for a direct comparison of results that can be interpreted in terms of electromagnetic parameters and thereby linked to composition.

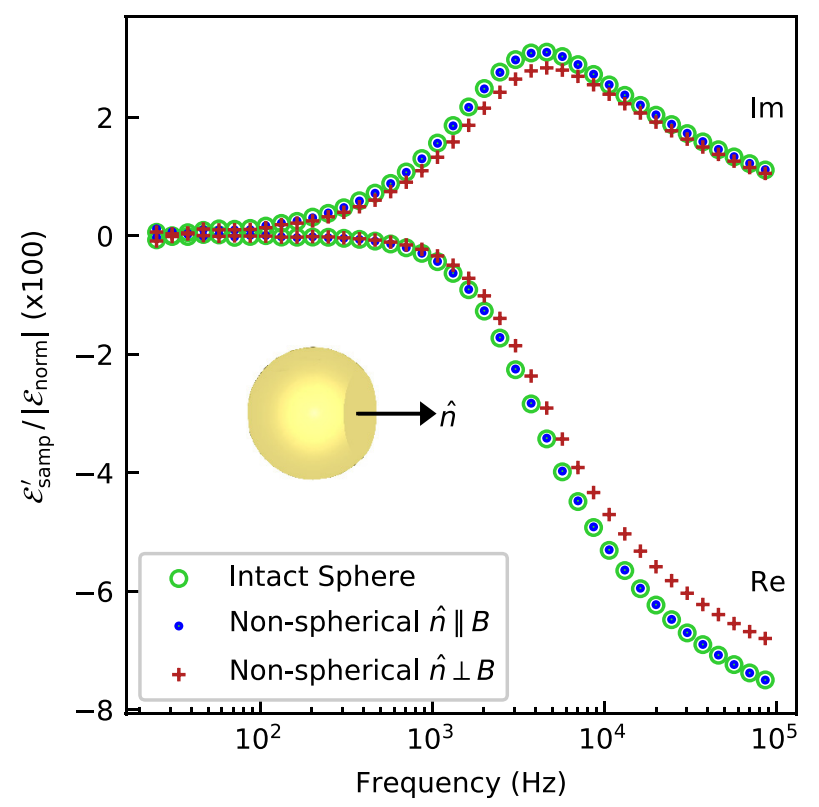

Fig. 8. Response of the spherical and non-spherical brass samples. The inset shows a drawing of the latter indicating the normal $\hat{\boldsymbol{n}}$ to the flat faces. For clarity only every fifth data point is shown. 
The results of the tests are shown in Fig. 9. There are three distinct response curves corresponding to each of the three possible compositions. Three of the coins (1996, 1999, and 2003-1) exhibit vanishing signal in the limit $f \rightarrow 0$, a clear signature that they are non-magnetic. Of these three, the 1996 coin approaches the asymptotic behavior of the thick limit (i.e., complete flux expulsion with eddy currents confined to a vanishingly small skin depth) at a lower frequency than the others, which reflects the higher electrical conductivity of copper versus zinc. The other two non-magnetic coins have identical response curves and must therefore be copper plated zinc. The fourth coin (2003-2) exhibits the clear hallmarks of a magnetic metal: it has a non-zero real component in the dc limit, and the thick limit does not appear until higher frequency. The relative features of the electromagnetic response of all four coins are clearly distinguishable and are consistent with their possible compositions based on date. As such, this method demonstrates the use of non-destructive testing for identifying and sorting visually similar metallic objects.

\section{USING A FUNCTION GENERATOR AND OSCILLOSCOPE INSTEAD OF A LOCK-IN AMPLIFIER}

While the lock-in amplifier is a standard device for most research laboratories, it may be a luxury in the undergraduate teaching laboratory. In recognition of this, we demonstrate that it is possible to run this experiment successfully using a function generator and oscilloscope in lieu of the lock-in. We happened to use an Agilent 33210A waveform generator and an Agilent 2000 series oscilloscope. The most important considerations, however, are that both devices can be computer controlled and that the oscilloscope has multiple inputs and can provide a difference measurement of two of its channels.

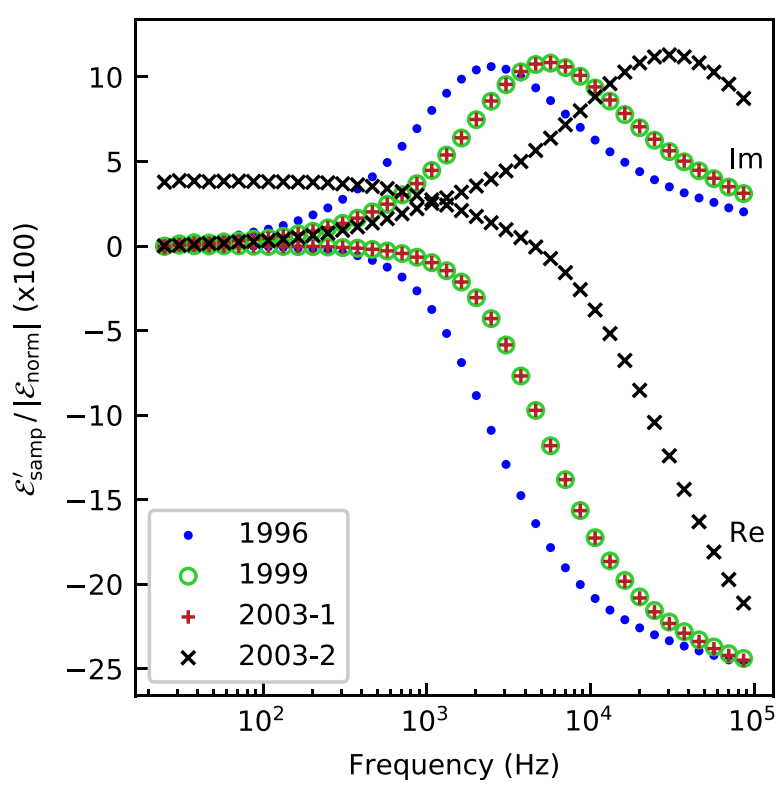

Fig. 9. Response of 1-cent coins with $\boldsymbol{B}(t)$ applied normal to the coin face. For clarity only every fifth data point is shown. The year of minting and sample number are given in the legend. From a comparison of the response curves, one can deduce that coin 2003-1 is copper plated zinc and coin 2003-2 is copper plated steel/nickel.
In this configuration of the experiment, the functiongenerator output is used to drive the Helmholtz coil and the synchronized output is used to trigger the oscilloscope via one of its input channels. The pick-up coils are connected to two of the other oscilloscope channels. After each change in frequency, the computer program adjusts the sensitivity and time base of the oscilloscope if necessary, then records the peak-to-peak voltage of the difference measurement, as well as its phase relative to the reference signal. In general, such data would be collected for the normalization, background, and foreground run as described above, and the same signal processing steps would be performed to determine $\mathcal{E}_{\text {samp }}^{\prime} /\left|\mathcal{E}_{\text {norm }}\right|$. However, we found that the direct coupling between the Helmholtz and pick-up coils could not be resolved by the oscilloscope, so the background run and subtraction were not done here.

An example of data collected with this method using the aluminum sphere is shown in Fig. 10. The signal-to-noise ratio is clearly not as good as with the lock-in amplifier. We also found that at frequencies below $1000 \mathrm{~Hz}$, the signal was too weak for the oscilloscope to make meaningful phase measurements, and as a result, data were not collected in this range. To best compare with the results in Fig. 5, the upper limit of the frequency range was kept to $102 \mathrm{kHz}$. A fit of Eq. (14) to the data in Fig. 10 gives $\sigma=20.55(7) \mathrm{MS} / \mathrm{m}$, which is consistent with the results of Sec. IV A, though not within error.

A possible advantage of this method, of course, is that frequencies higher than $102 \mathrm{kHz}$ can be explored. This would provide more data over a larger frequency range, which in turn could better constrain the fit parameters. One should be mindful, however, that a natural upper limit always occurs due to the self-resonance of the coils.

\section{CONCLUSION}

We have developed a very simple experiment to make precise measurements of the electromagnetic response of small metallic objects to a uniform ac magnetic field over a very

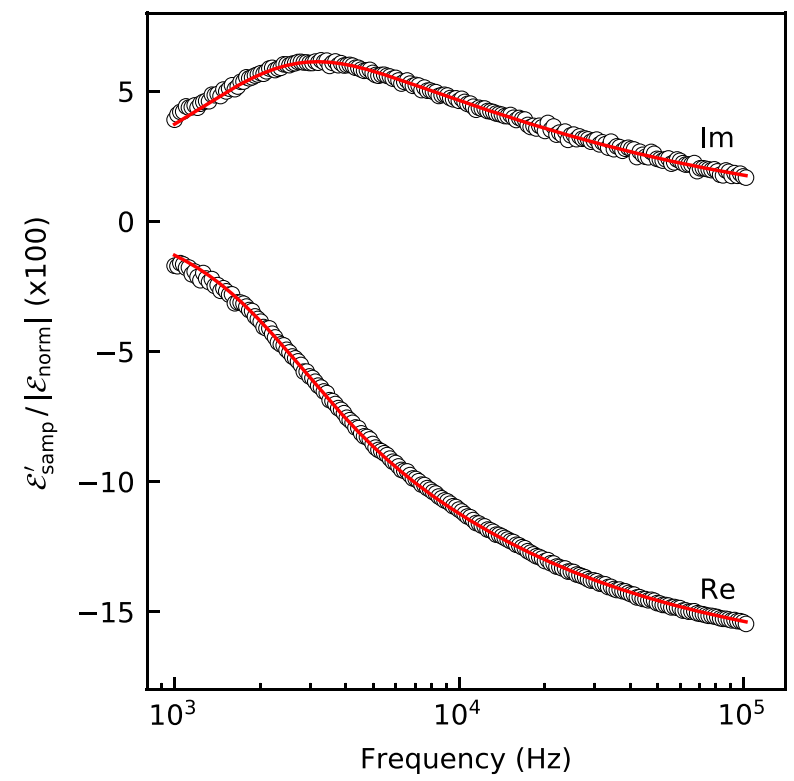

Fig. 10. The response of the aluminum sphere measured using a function generator and oscilloscope: data (circles), fit function for the non-magnetic model (solid lines). 
broad range of frequencies. Measurements on commercially available metal spheres fit very well to an exactly solvable model, allowing the simultaneous determination of the magnetic permeability and electrical conductivity given the sphere radius only. Modest efforts to characterize experimental error suggest that the precision and accuracy of these results is at the level of five parts per thousand or better. Qualitative experiments with non-spherical samples were used to demonstrate some of the uses and principles of eddycurrent-based non-destructive testing.

The work presented here leaves significant room for student exploration and innovation. For example, we made no attempt to optimize the pick-up coil design or location to maximize flux linkage with the sample. Nor did we attempt to develop new models that would incorporate nonlinear media, frequency-dependent material properties, or nonspherical samples. There are a multitude of sample shapes and materials that can be explored, of course, as well as many other uses of eddy-current-based non-destructive testing, such as crack detection, that can be tried.

\section{ACKNOWLEDGMENTS}

The authors thank J. Matwiy, D. Ostapchuk and M. Lang for their assistance constructing apparatus and writing code. The authors gratefully acknowledge the support of the Natural Sciences and Engineering Research Council of Canada, especially the Undergraduate Student Research Award for MLH.

${ }^{\text {a)} E l e c t r o n i c ~ m a i l: ~ m l h o n k e @ u w a t e r l o o . c a ; ~ P r e s e n t ~ a d d r e s s: ~ C h e r i t o n ~}$ School of Computer Science, University of Waterloo, N2L 3G1, Waterloo, Ontario, Canada.

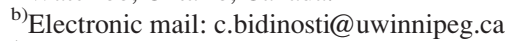

${ }^{1}$ D. J. Griffiths, Introduction to Electrodynamics, 4th ed. (Pearson, Boston, 2013), pp. 412-414.

${ }^{2}$ J. D. Jackson, Classical Electrodynamics, 2nd ed. (John Wiley \& Sons, New York, 1975), pp. 296-298.

${ }^{3}$ W. R. Smythe, Static and Dynamic Electricity, 2nd ed. (McGraw-Hill, New York, 1950), Chap. XI.

${ }^{4}$ L. D. Landau, E. M. Lifshitz, and L. P. Pitaevskii, Electrodynamics of Continuous Media, 2nd ed. (Pergamon Press, Oxford, 1984), Chap. VII.

${ }^{5}$ A. Garg, Classical Electromagnetism in a Nutshell (Princeton U.P., Princeton, 2012), Chaps. 16, 18, and 19.

${ }^{6} \mathrm{H}$. Lamb, "On electrical motions in a spherical conductor," Philos. Trans. R. Soc. London 174, 519-549 (1883).

${ }^{7}$ L. V. King, "XXI. Electromagnetic shielding at radio frequencies," Philos. Mag. Ser. 7 15, 201-223 (1933).

${ }^{8}$ J. R. Wait, "A conducting sphere in a time varying magnetic field," Geophysics 16, 666-672 (1951).

${ }^{9}$ M. P. Perry and T. B. Jones, "Eddy current induction in a solid conducting cylinder with a transverse magnetic field," IEEE Trans. Magn. 14, 227-232 (1978).

${ }^{10}$ G. Wouch and A. E. Lord, Jr., "Eddy currents: Levitation, metal detectors, and induction heating," Am. J. Phys. 46, 464-466 (1978).

${ }^{11} \mathrm{~S}$. Fahy et al., "Electromagnetic screening by metals," Am. J. Phys. 56, 989-992 (1988).
${ }^{12}$ C. P. Bidinosti et al., "The sphere in a uniform rf field-revisited," Con. Mag. Reson. 31B, 191-202 (2007).

${ }^{13}$ A. Garg, "Conductors in quasistatic electric fields," Am. J. Phys. 76, 615-620 (2008).

${ }^{14} \mathrm{~J}$. Íñiguez et al., "The electromagnetic field in conductive slabs and cylinders submitted to a harmonic longitudinal magnetic field," Am. J. Phys. 77, 1074-1081 (2009).

${ }^{15}$ M. E. Hayden et al., "Specific absorption rates and signal-to-noise ratio limitations for MRI in very-low magnetic fields," Con. Mag. Reson. 40A, 281-294 (2012)

${ }^{16} \mathrm{~L}$. Zilberti et al., "The underestimated role of gradient coils in MRI safety," Mag. Reson. Med. 77, 13-15 (2017).

${ }^{17}$ M. D. O'Toole et al., "Non-contact multi-frequency magnetic induction spectroscopy system for industrial-scale bio-impedance measurement," Meas. Sci. Technol. 26, 035102 (2015).

${ }^{18}$ M. A. Nurge et al., "A thick-walled sphere rotating in a uniform magnetic field: The next step to de-spin a space object," Am. J. Phys. 85, 596-610 (2017).

${ }^{19} \mathrm{H}$. Braunisch et al., "Magnetoquasistatic response of conducting and permeable prolate spheroid under axial excitation," IEEE Trans. Geosci. Remote Sensing 39, 2689-2701 (2001).

${ }^{20} \mathrm{Y}$. Das et al., "Time domain response of a sphere in the field of a coil: Theory and experiment," IEEE Trans. Geosci. Remote Sensing 22, 360-367 (1984).

${ }^{21}$ Y. He et al., "Pulsed eddy current technique for defect detection in aircraft riveted structures," Nondestruc. Testing Eval. Int. 43, 176-181 (2010).

${ }^{22}$ R. Pohl et al., "NDT techniques for railroad wheel and gauge corner inspection," Nondestruc. Testing Eval. Int. 37, 89-94 (2004).

${ }^{23}$ W. M. Saslow, Electricity, Magnetism, and Light (Academic Press, Boston, 2002), Chaps. 9, 13, and 14.

${ }^{24}$ W. M. Saslow, "How a superconductor supports a magnet, how magnetically soft iron attracts a magnet, and eddy currents for the uninitiated," Am. J. Phys. 59, 16-25 (1991).

${ }^{25}$ W. M. Saslow, "Maxwell's theory of eddy currents in thin conducting sheets, and applications to electromagnetic shielding and MAGLEV," Am. J. Phys. 60, 693-711 (1992).

${ }^{26} \mathrm{~J}$. R. Gosselin et al., "Study of eddy currents in a cylindrical wire: an undergraduate laboratory experiment," Am. J. Phys. 50, 440-443 (1982).

${ }^{27}$ Y. Kraftmakher, "Eddy currents: Contactless measurement of electrical resistivity," Am. J. Phys. 68, 375-379 (2000).

${ }^{28} \mathrm{~J}$. Íñiguez et al., "Measurement of electrical conductivity in nonferromagnetic tubes and rods at low frequencies," Am. J. Phys. 77, 949-953 (2009).

${ }^{29}$ N. W. Ashcroft and N. D. Mermin, Solid State Physics (Saunders College Publishing, Fort Worth, 1976), Chaps. 1, 31, and 33.

${ }^{30}$ E. M. Purcell, Electricity and Magnetism, 2nd ed. (Cambridge U.P., Cambridge, 2011), Chap. 11.

${ }^{31}$ W. M. Haynes, D. R. Lide, and T. J. Bruno, CRC Handbook of Chemistry and Physics, 97th ed. (CRC Press, Boca Raton, 2017).

${ }^{32}$ J. R. Davis, ASM Specialty Handbook: Aluminum and Aluminum Alloys (ASM International, Materials Park, OH, 1993), p. 68.

${ }^{33}$ C. R. Paul, Introduction to Electromagnetic Compatibility, 2nd ed. (John Wiley \& Sons, Hoboken NJ, 2006), p. 21.

${ }^{34}$ Wikipedia, Tungsten Carbide: < https://en.wikipedia.org/wiki/ Tungsten_carbide $>$.

${ }^{35}$ J. R. Davis, ASM Specialty Handbook: Stainless Steels (ASM International, Materials Park, OH, 1994), p. 10.

${ }^{36} \mathrm{~N}$. Bowler, "Frequency-dependence of relative permeability in steel," AIP Conf. Proc. 820, 1269-1276 (2006).

${ }^{37}$ The technical specifications of the Canadian 1-cent coin throughout its history can presently be found online here: www.mint.ca/store/mint/aboutthe-mint/1-cent-5300004. 